\title{
TIMELIKE MINIMAL SUBMANIFOLDS OF GENERAL CO-DIMENSION IN MINKOWSKI SPACETIME
}

\author{
PAUL ALLEN, LARS ANDERSSON, JAMES ISENBERG
}

\begin{abstract}
We consider the timelike minimal surface problem in Minkowski spacetimes and show local and global existence of such surfaces having arbitrary dimension $\geq 2$ and arbitrary co-dimension, provided they are initially close to a flat plane.
\end{abstract}

\section{INTRODUCTION}

In this work we consider timelike minimal submanifolds of dimension $1+n$, $n \geq 2$, of Minkowski spacetimes of dimension $1+n+q, q \geq 1$. A submanifold is called minimal if it is stationary with respect to variations of the induced area, which thus provides an action for the system. Timelike minimal submanifolds may be viewed as simple but nontrivial examples of D-branes, which play an important role in string-theory, and the system under consideration here thus has natural generalizations motivated by string theory. In this work we prove a small data, global existence result for timelike minimal submanifolds of arbitrary codimension $q$. The solutions which are constructed are close to flat timelike planes.

The Euler-Lagrange equations arising from variation of the area form a quasilinear system of PDE's, which under suitable conditions on the data is hyperbolic. This system is closely related to the scalar quasilinear hyperbolic PDE governing timelike minimal hypersurfaces. The small data, global existence problem for timelike minimal hypersurfaces has been considered by Brendle $\mathrm{B}$ and Lindblad [1]. The work of Lindblad makes use of the null structure of the system, and our approach is closely related to the work in L1].

Consider an embedding of $\mathbb{R}^{1+n}$ into Minkowski spacetime $\mathbb{R}^{1+n+q}$ given by the graph of a map $f: \mathbb{R}^{1+n} \rightarrow \mathbb{R}^{q}$. Let greek indices $\alpha, \beta, \ldots$ take values in $0,1, \ldots, n$ and let uppercase latin indices $I, J, \ldots$ take values in $1, \ldots, q$. We introduce cartesian coordinates $x^{\alpha}$ on $\mathbb{R}^{1+n}$ and $x^{I}$ on $\mathbb{R}^{q}$. The induced metric $\mathbb{R}^{1+n}$ is

$$
h_{\alpha \beta}=\eta_{\alpha \beta}+f_{\alpha}^{I} f_{\beta}^{J} \delta_{I J},
$$

where $f^{I}=x^{I} \circ f, f_{\alpha}^{I}=\partial_{\alpha} f^{I}$ and $\eta=\operatorname{diag}(-1,1 \ldots, 1)$ is the Minkowski metric. Varying the action

$$
\mathcal{S}=\int \sqrt{-\operatorname{det} h_{\mu \nu}} d^{1+n} x,
$$

yields the Euler-Lagrange equations

$$
0=\partial_{\mu}\left[\sqrt{-\operatorname{det} h} h^{\mu \nu} f_{\nu}^{I}\right] \quad I=1, \ldots, q,
$$

Date: 1 December 2005. 
which we consider for small data

$$
f^{I}(0, \cdot)=\varepsilon g^{I} \quad \partial_{t} f^{I}(0, \cdot)=\varepsilon k^{I},
$$

with $g^{I}, k^{I}$ smooth and decaying sufficiently fast for large $|x|$; for simplicity we restrict to $\varepsilon \leq 1$. Here $h^{\mu \nu}$ is the inverse of $h_{\alpha \beta}$.

For future use, we note that equation (1.2) can be written in divergence form

$$
\square f^{I}=\partial_{\mu}\left[F^{\mu \nu} f_{\nu}^{I}\right]
$$

where $\square=\eta^{\mu \nu} \partial_{\mu} \partial_{\nu}$ is the Minkowski wave operator and $F^{\mu \nu}(\partial f)=\eta^{\mu \nu}-\sqrt{-\operatorname{det} h} h^{\mu \nu}$, as well as in the form

$$
H_{J L}^{\mu \nu}(\partial f) \partial_{\mu} \partial_{\nu} f^{J}=0, \quad I=1, \ldots, q
$$

where

$H_{J L}^{\mu \nu}=\sqrt{-\operatorname{det} h}\left[\delta_{J L} h^{\mu \nu}-\delta_{I J} \delta_{K L}\left(h^{\mu \nu} h^{\alpha \beta} f_{\alpha}^{K} f_{\beta}^{I}+h^{\mu \alpha} h^{\nu \beta} f_{\alpha}^{I} f_{\beta}^{K}+h^{\mu \alpha} h^{\nu \beta} f_{\alpha}^{K} f_{\beta}^{I}\right)\right]$.

We raise and lower Greek (intrinsic) indices using $h_{\mu \nu}$ and its inverse, while Latin (extrinsic) indices are raised and lowered using the identity $\delta_{I J}$ and its inverse. From equation (1.6) it follows that $H_{J L}^{\mu \nu}$ has the symmetries

$$
H_{J L}^{\mu \nu}=H_{L J}^{\mu \nu}=H_{J L}^{\nu \mu} .
$$

Due to the symmetries, an energy estimate and local well posedness holds for the system (1.5).

The local existence argument follows [H1] (see also [S] ) and uses an energy inequality which takes advantage of symmetries in the system; see KSS for a treatment of well-posedness and lifespan results for symmetric systems. The global existence result also requires estimates applicable to divergence equations (see [L2]) and an $L^{\infty}-L^{1}$ estimate. Furthermore, the global existence result in $n=2$ dimension exploits the fact that the equation satisfies the null condition; see Section 4 for details.

\section{Local Existence}

The local well-posedness for systems of the form (1.5) is well established. Therefore we do not give a complete proof here but rather provide a simple proof of the basic energy estimate. The proof of local well-posedness then follows along the same lines as the proofs given in $[\mathrm{H} 1$ or $[\mathrm{S}$. Note that the energy estimate we state here also plays a key role in the global existence results discussed in the next section.

Write $|\partial f|^{2}=\eta^{\mu \nu} \delta_{I J} f_{\mu}^{I} f_{\nu}^{J}$; the function space norms used below are defined in terms of this and analogous expressions. Using the identity

$$
\begin{aligned}
\left(f_{0}^{I}\right)\left(H_{I J}^{\mu \nu} f_{\mu \nu}^{J}\right)=\partial_{\mu}\left[H^{\mu \nu}{ }_{I J} f_{\nu}^{I} f_{0}^{J}-\frac{1}{2}\right. & \left.H_{I J}^{\alpha \beta} f_{\alpha}^{I} f_{\beta}^{J} \delta_{0}^{\mu}\right] \\
& -\partial_{\mu}\left[H^{\mu \nu}{ }_{I J}\right] f_{\nu}^{I} f_{0}^{J}+\frac{1}{2} \partial_{0}\left[H^{\mu \nu}{ }_{I J}\right] f_{\mu}^{I} f_{\nu}^{J},
\end{aligned}
$$

a standard argument yields the following energy estimate. 
Lemma 1. Let $f \in C^{2}\left([0, T) \times \mathbb{R}^{n} ; \mathbb{R}^{q}\right)$ for $T>0$ and assume that $H^{\mu \nu}$ has the symmetries (1.7). Further, assume that $H_{I J}^{\mu \nu}$ satisfies

$$
\sum\left|H_{I J}^{\mu \nu}-\eta^{\mu \nu} \delta_{I J}\right|<\frac{1}{2} \quad \text { on }[0, T] .
$$

Then for $t \in[0, T)$ we have

$$
\begin{aligned}
\|\partial f(t, \cdot)\|_{L^{2}} \leq 2\left(\|\partial f(0, \cdot)\|_{L^{2}}+\int_{0}^{t} \sum_{I}\left\|H_{I J}^{\mu \nu} \partial_{\mu} \partial_{\nu} f^{J}(\tau, \cdot)\right\|_{L^{2}} d \tau\right) \\
\times \exp \left(\int_{0}^{t} 2\|\partial H(s, \cdot)\|_{L^{\infty}} d s\right) .
\end{aligned}
$$

With this energy estimate, the proof of local well-posedness can now be completed by an iteration procedure following exactly the outline in [S] or [H1]. The approach in H1 makes use of some extra structural assumptions which are easily removed, and gives local well-posedness in Sobolev spaces $H^{s}$, for integer $s>n / 2+2$. The argument in Sogge gives the result for $s>n+3$. Since we are concerned with small data, global existence here, the exact regularity needed for the local wellposedness is not important. We can now state the following result.

Theorem 1. Let $s>n / 2+2$ and consider equation

$$
H_{J L}^{\mu \nu}(x, f, \partial f) \partial_{\mu} \partial_{\nu} f^{J}=G_{L}(x, f, \partial f), \quad I=1, \ldots, q
$$

with initial data

$$
f(0, \cdot)=g, \quad \partial_{0} f(0, \cdot)=k
$$

Here $H_{J L}^{\mu \nu}$ and $G_{L}$ are assumed to be smooth functions of their arguments and $H_{J L}^{\mu \nu}$ is assumed to satisfy the symmetries (1.7). Suppose the initial data $(g, k)$ is such that equation (2.1) is valid for $H^{\mu \nu}{ }_{J L}$ evaluated on $(g, k)$. Then there is a $T>0$, which depends only on the norm of $(g, k)$ in $H^{s} \times H^{s-1}$, and a function $f \in C^{2}\left([0, T] \times \mathbb{R}^{n} ; \mathbb{R}^{q}\right)$ which solves (2.3), with $\left|\partial^{\alpha} f\right|$ bounded for $|\alpha| \leq 2$. The maximal time of existence is bounded from below by the supremum of all $T$ such that (2.3) has a $C^{2}$ solution such that for $0 \leq t \leq T$, equation (2.1) is valid and $\partial^{\alpha} f$ is bounded for $|\alpha| \leq 2$.

\section{Global existence in Dimensions $n \geq 3$}

Global existence follows from a procedure similar to that discussed in [L1. The estimates needed rely on a collection of weighted norms defined using the set of Lorentz vector fields $Z_{\mu}$, which include the generators of the Lorentz group, along with the generator of dilations:

$$
\left\{\partial_{\mu}, \Omega_{a b}:=x^{b} \partial_{a}-x^{a} \partial_{b}, \Omega_{0 a}:=t \partial_{a}+x^{a} \partial_{t}, L:=t \partial_{t}+r \partial_{r}\right\} .
$$

These form a Lie algebra, which satisfies the following commutation relations

$$
\left[Z_{\mu}, \partial_{\nu}\right]=\sum a_{\mu \nu}{ }^{\alpha} \partial_{\alpha}, \quad a_{\mu \nu}{ }^{\alpha}=0, \pm 1, \quad \text { and } \quad\left[Z_{\mu}, \square\right]= \begin{cases}-2 \square & \text { if } Z_{\mu}=L \\ 0 & \text { otherwise }\end{cases}
$$

It follows that the equation $\square \psi=0$ is preserved by these operators. We now define the following norms in terms of products $Z^{\alpha}$ (for multi-index $\alpha$ ) of the Lorentz 
vector fields applied to $f$ :

$$
\begin{aligned}
M_{1}(t) & \equiv \sum_{|\alpha| \leq m}\left\|\partial Z^{\alpha} f(t, \cdot)\right\|_{L^{2}}, \\
M_{2}(t) & \equiv \sum_{|\alpha| \leq m}\left\|Z^{\alpha} f(t, \cdot)\right\|_{L^{2}}, \\
N_{1}(t) & \equiv \sum_{|\alpha| \leq l}\left\|\partial Z^{\alpha} f(t, \cdot)\right\|_{L^{\infty}}, \\
N_{2}(t) & \equiv \sum_{|\alpha| \leq l+1}\left\|Z^{\alpha} f(t, \cdot)\right\|_{L^{\infty}},
\end{aligned}
$$

Here $m$ is an integer such that $m>2 n+1$ and $l=(m+1) / 2$. Since $\partial_{\mu} \in\left\{Z_{\nu}\right\}$, these norms control the $L^{2}$ and $L^{\infty}$ Sobolev norms (of order $k$ and $l$, respectively) of $f$ and $\partial f$. It follows that controlling these norms is sufficient to overcome the obstruction to local existence discussed at the end of the previous section. In particular, if we control these norms, then $f$ is bounded in $C^{2}$. Furthermore, we use the norms $N_{1}, N_{2}$ to show stability in the sense that they decay as $t$ grows. Note that any estimate for $N_{2}$ implies an estimate for $N_{1}$ as well.

The following three propositions also play a role in our proof of global existence. (See [L2], and also [L1, [H2], [K1], for some of the proofs of these results.)

Proposition 1. If $g$ is a solution to

$$
\left\{\begin{array}{l}
\square g=\partial_{\mu} F^{\mu} \\
g(0, \cdot)=\varepsilon g, \quad \partial_{0} g(0, \cdot)=\varepsilon k
\end{array}\right.
$$

then

$$
\|g(t, \cdot)\|_{L^{2}} \leq C_{d a t a} \varepsilon m(t)+\sum_{\mu} \int_{0}^{t}\left\|F^{\mu}(\tau, \cdot)\right\|_{L^{2}} d \tau
$$

where

$$
m(t)= \begin{cases}\log (2+t) & \text { if } n=2, \\ 1 & \text { otherwise, }\end{cases}
$$

and $C_{d a t a}$ depends on $g, k$, and $F^{0}(0, \cdot)$.

Proposition 2. The solution to

$$
\left\{\begin{array}{l}
\square g=G \\
g(0, \cdot)=\varepsilon g_{0}, \quad \partial_{0} g(0, \cdot)=\varepsilon g_{1}
\end{array}\right.
$$

satisfies

$$
|g(t, x)| \leq \frac{C}{(1+t+|x|)^{(n-1) / 2}}\left(C_{d a t a} \varepsilon+\int_{0}^{t} \sum_{|\alpha| \leq n-1}\left\|\frac{Z^{\alpha} G}{(1+s+|\cdot|)^{(n-1) / 2}}(s, \cdot)\right\|_{L^{1}} d s\right) .
$$


To make use of the above propositions in controlling the norms (3.1), we apply $Z^{\alpha}$ to both sides of the equation (1.5) for $f^{I}$, and obtain

$$
H_{I J}^{\mu \nu} \partial_{\mu} \partial_{\nu}\left(Z^{\alpha} f^{J}\right)=\sum_{k \geq 3, \sum\left|\alpha_{i}\right| \leq|\alpha|+1} H_{I, I_{1} \cdots I_{k}, \gamma_{1} \cdots \gamma_{k}, \alpha_{1} \cdots \alpha_{k}}\left(\partial_{\gamma_{1}} Z^{\alpha_{1}} f^{I_{1}}\right) \cdots\left(\partial_{\gamma_{k}} Z^{\alpha_{k}} f^{I_{k}}\right) .
$$

Since $h^{\mu \nu}=\eta^{\mu \nu}+\mathcal{O}\left(|\partial f|^{2}\right)$, this may also be written as

$$
\square\left(Z^{\alpha} f^{I}\right)=\sum_{k \geq 3, \sum\left|\alpha_{i}\right| \leq|\alpha|+1} \hat{H}_{I_{1} \cdots I_{k}, \gamma_{1} \cdots \gamma_{k}, \alpha_{1} \cdots \alpha_{k}}^{I}\left(\partial_{\gamma_{1}} Z^{\alpha_{1}} f^{I_{1}}\right) \cdots\left(\partial_{\gamma_{k}} Z^{\alpha_{k}} f^{I_{k}}\right) .
$$

with some modified coefficient functions $\hat{H}$, satisfying $\hat{H}=\mathcal{O}\left(|\partial f|^{2}\right)$. Note that at most one of the $\alpha_{i}$ can satisfy $\left|\alpha_{i}\right|>(|\alpha|+1) / 2$. The global existence proof also depends on the form of the divergence equation (1.4). In particular, we note that $\sqrt{\operatorname{det}[h]} h^{\mu \nu}=\eta^{\mu \nu}+\mathcal{O}\left(|\partial f|^{2}\right)$ as $|\partial f| \rightarrow 0$, and thus $F^{\mu \nu}:=\eta^{\mu \nu}-\sqrt{-\operatorname{det} h} h^{\mu \nu}=$ $\mathcal{O}\left(|\partial f|^{2}\right)$. Hence applying $Z^{\alpha}$ to (1.4) we obtain

$$
\square\left(Z^{\alpha} f^{I}\right)=\partial_{\mu}\left[\sum_{k \geq 3, \sum\left|\alpha_{i}\right| \leq|\alpha|} F_{I_{1} \cdots I_{k}, \gamma_{1} \cdots \gamma_{k}, \alpha_{1} \cdots \alpha_{k}}^{\mu, I}\left(\partial_{\gamma_{1}} Z^{\alpha_{1}} f^{I_{1}}\right) \cdots\left(\partial_{\gamma_{k}} Z^{\alpha_{k}} f^{I_{k}}\right)\right] .
$$

where again at most one of the $\alpha_{i}$ can satisfy $\left|\alpha_{i}\right|>|\alpha| / 2$.

We are now prepared to show global existence in dimensions $n \geq 3$. The proof uses a continuous induction, or bootstrap argument.

To set up the bootstrap argument, we assume that there is a constant $K$ so that on $[0, T)$ we have the following estimates for the norms defined in (3.1):

$$
\begin{aligned}
M_{1}(t) & \leq K \varepsilon, \\
M_{2}(t) & \leq K \varepsilon, \\
N_{1}(t) & \leq \frac{K \varepsilon}{(1+t)^{\frac{n-1}{2}}}, \\
N_{2}(t) & \leq \frac{K \varepsilon}{(1+t)^{\frac{n-1}{2}}} .
\end{aligned}
$$

To close the bootstrap, we show that we can in fact choose $K$ sufficiently large and $\epsilon$ sufficiently small so that the above inequalities hold independently of $T$ with $K \varepsilon$ replaced by $K \varepsilon / 2$. This implies that for small data, solutions can be extended for all $T>0$.

Applying the energy estimate of Lemma 10 (3.2) and summing over $|\alpha| \leq k$, we have

$$
M_{1}(t) \leq C\left(C_{\mathrm{data}} \varepsilon+\int_{0}^{t} C_{N_{1}} N_{1}(s)^{2} M_{1}(s) d s\right) \cdot \exp \left(C \int_{0}^{t} N_{1}(\tau)^{2} d \tau\right),
$$

where $C_{N_{1}}$ is a constant absorbing possible "extra" factors of $N_{1}(s)$ and only reflects the finiteness of $N_{1}(s)$. Likewise, applying Proposition 1 to (3.4) and Proposition 2 to (3.3) gives us

$$
M_{2}(t) \leq C \varepsilon+\int_{0}^{t} C_{N_{1}} N_{1}(s)^{2} M_{1}(s) d s
$$


and

$$
N_{2}(t) \leq \frac{C}{(1+t)^{(n-1) / 2}}\left(C_{\text {data }} \varepsilon+\int_{0}^{t} \frac{\left(N_{1}(s)+N_{2}(s)\right)}{(1+s)^{(n-1) / 2}}\left(M_{1}(s)+M_{2}(s)\right)^{2} d s\right)
$$

where we have made use of the Cauchy-Schwartz inequality. Under the assumed bounds, we have that for some $C$,

$$
\begin{aligned}
& M_{1}(t) \leq e^{C(K \varepsilon)^{2}}\left(C_{\text {data }} \varepsilon+C(K \varepsilon)^{2} K \varepsilon\right) \leq \frac{K \varepsilon}{2}, \\
& M_{2}(t) \leq C_{\text {data }} \varepsilon+C(K \varepsilon)^{2} K \varepsilon \leq \frac{K \varepsilon}{2} \\
& N_{2}(t) \leq \frac{1}{(1+t)^{(n-1) / 2}}\left(C C_{\text {data }} \varepsilon+C(K \varepsilon)^{4}\right) \leq \frac{K \varepsilon}{2(1+t)^{(n-1) / 2}},
\end{aligned}
$$

where the second inequality in each line holds for all $t$ if $K$ is chosen sufficiently large and $\varepsilon$ is chosen sufficiently small. Recall that an estimate for $N_{1}(t)$ follows from the estimate for $N_{2}(t)$. Obtaining these tighter bounds on the norms, we have closed the bootstrap. In view of the continuation property stated in Theorem 1 . we have proved small data global existence for $n \geq 3$.

\section{Global existence in Dimension $n=2$}

In the case of $n=2$, we require more detailed information concerning the structure of the system. In particular, we exploit the fact that the system satisfies the so-called "null-condition" of Klainerman K2, K3], which is a condition on the quadratic part of the nonlinearity. We consider null forms, quadradic forms of first derivatives, which are given by

$$
Q_{00}(f, g)=\eta^{\mu \nu}\left(\partial_{\mu} f\right)\left(\partial_{\nu} g\right), \quad Q_{\alpha \beta}(f, g)=\left(\partial_{\alpha} f\right)\left(\partial_{\beta} g\right)-\left(\partial_{\beta} f\right)\left(\partial_{\alpha} g\right), \quad \alpha \neq \beta
$$

and satisfy time decay closer to that of cubic terms. In particular, if $Q$ is any null form, then

$$
|Q(f, g)(t, x)| \leq \frac{C}{1+t+|x|} \sum_{|\alpha|=1}\left|Z^{\alpha} f(t, x)\right| \sum_{|\beta|=1}\left|Z^{\beta} g(t, x)\right| .
$$

Furthermore, for any Lorentz vector field $Z$ and null form $Q$ there exists constants $a^{\mu \nu}$ so that

$$
Z Q(f, g)=Q(Z f, g)+Q(f, Z g)+a^{\mu \nu} Q_{\mu \nu}(f, g) .
$$

Returning to the system (1.2), we note that the Lagrangian associated to the volume element of the induced metric is $\mathcal{L}=\sqrt{-\operatorname{det} h}$. For small $|\partial f|$, we have

$$
\begin{aligned}
-\operatorname{det} h & =1+\eta^{\mu \nu} \delta_{I J} f_{\mu}^{I} f_{\nu}^{J}+\mathcal{O}\left(|\partial f|^{4}\right) \\
& =1+\delta_{I J} Q_{00}\left(f^{I}, f^{J}\right)+\mathcal{O}\left(|\partial f|^{4}\right)
\end{aligned}
$$

and thus the Euler-Lagrange equations take the form

$$
\left(1+\delta_{K L} Q_{00}\left(f^{K}, f^{L}\right)\right) \square f^{J}=\frac{1}{2} \eta^{\mu \nu} f_{\mu}^{J} \partial_{\nu}\left[\delta_{A B} Q_{00}\left(f^{A}, f^{B}\right)\right]+\mathcal{O}\left(\left|\partial^{2} f\right| \cdot|\partial f|^{4}\right) \text {. }
$$

For small $|\partial f|$ we have that

$$
\left(1+\delta_{K L} Q_{00}\left(f^{K}, f^{L}\right)\right)^{-1}=1+\mathcal{O}\left(|\partial f|^{2}\right)
$$


thus we obtain

$$
\square f^{J}=\frac{1}{2} Q_{00}\left(f^{J}, \delta_{A B} Q_{00}\left(f^{A}, f^{B}\right)\right)+\mathcal{O}\left(\left|\partial^{2} f\right| \cdot|\partial f|^{4}\right) .
$$

Applying $Z^{\alpha}$ yields

$$
\square\left(Z^{\alpha} f^{J}\right)=\sum_{\sum\left|\alpha_{i}\right| \leq|\alpha|} Q_{00}\left(Z^{\alpha_{1}} f^{J}, \delta_{A B} Q_{00}\left(Z^{\alpha_{2}} f^{A}, Z^{\alpha_{3}} f^{B)}\right)+\mathcal{O}\left(\left|Z^{\beta_{1}} \partial^{2} f\right| \cdot\left|Z^{\beta_{2}}(\partial f)^{4}\right|\right),\right.
$$

where $\left|\alpha_{1}\right|+\left|\beta_{2}\right| \leq|\alpha|$.

The proof of global existence when $n=2$ follows closely the proof for higher dimensions, with three differences. First, the null equation (4.7) is used in place of (3.3). Second, we use the following variation of the energy estimate of Lemma 1 .

Lemma 2. Under the hypotheses of Lemma 1 we have

$$
\begin{aligned}
\|\partial f(t, \cdot)\|_{L^{2}} \leq & C\|\partial f(0, \cdot)\|_{L^{2}} \cdot \exp \left(-\int_{0}^{t} C\|\partial H(\tau, \cdot)\|_{L^{\infty}} d \tau\right) \\
& +C \int_{0}^{t} \sum_{I}\left\|B_{I}(s, \cdot)\right\|_{L^{2}} \cdot \exp \left(-\int_{s}^{t} C\|\partial H(\tau, \cdot)\|_{L^{\infty}} d \tau\right) d s .
\end{aligned}
$$

The third difference is that the bootstrap assumptions of (3.5) are replaced by the following:

$$
\begin{aligned}
& M_{1}(t) \leq K \varepsilon(1+t)^{\delta}, \\
& M_{2}(t) \leq K \varepsilon(1+t)^{\delta}, \\
& N_{1}(t) \leq \frac{K \varepsilon}{(1+t)^{1 / 2}}, \\
& N_{2}(t) \leq \frac{K \varepsilon}{(1+t)^{1 / 2}},
\end{aligned}
$$

where $0<\delta<\frac{1}{2}$ is a fixed, arbitrary constant. We apply Lemma 2 to (3.2) and obtain the estimate

$$
M_{1}(t) \leq C_{\text {data }} \varepsilon \cdot \exp \left(\int_{0}^{t} \frac{C(K \varepsilon)^{2}}{(1+\tau)} d \tau\right)+\int_{0}^{t} \frac{C(K \varepsilon)^{3}}{(1+s)^{1-\delta}} \cdot \exp \left(\int_{s}^{t} \frac{C(K \varepsilon)^{2}}{(1+\tau)} d \tau\right) d s .
$$

Computing

$$
\exp \left(\int_{s}^{t} \frac{C(K \varepsilon)^{2}}{(1+\tau)} d \tau\right)=\exp \left(C(K \varepsilon)^{2} \log \left(\frac{1+t}{1+s}\right)\right)=\left(\frac{1+t}{1+s}\right)^{C(K \varepsilon)^{2}},
$$

one sees that

$$
\begin{aligned}
M_{1}(t) & \leq C_{\text {data }} \varepsilon(1+t)^{C(K \varepsilon)^{2}}+C(K \varepsilon)^{3}(1+t)^{C(K \varepsilon)^{2}} \int_{0}^{t}(1+s)^{\delta-1-C(K \varepsilon)^{2}} d s \\
& =C_{\text {data }} \varepsilon(1+t)^{C(K \varepsilon)^{2}}+C(K \varepsilon)^{3}(1+t)^{C(K \varepsilon)^{2}}\left[(1+t)^{\delta-C(K \varepsilon)^{2}}-1\right] \\
& \leq K \varepsilon(1+t)^{\delta} / 2
\end{aligned}
$$


for large $K$ and small $\varepsilon$. Similarly, application of Proposition 1 to (3.4) implies that for suitable large $K$ and small $\varepsilon$ we have that

$$
\begin{aligned}
M_{2}(t) & \leq C_{\mathrm{data}} \varepsilon \log (2+t)+\int_{0}^{t} \frac{C(K \varepsilon)^{3}}{(1+s)^{1-\delta}} d s \\
& \leq C_{\mathrm{data}} \varepsilon \log (2+t)+\tilde{C}(K \varepsilon)^{3}(1+t)^{\delta} \leq(1+t)^{\delta} K \varepsilon / 2 .
\end{aligned}
$$

Lastly, via application of Proposition 2 to (4.7) and use of the null estimate (4.2) we obtain

$$
\begin{aligned}
N_{2}(t) & \leq \frac{C}{(1+t)^{1 / 2}}\left(C_{\mathrm{data}} \varepsilon+\int_{0}^{t} \frac{C(K \varepsilon)^{3}}{(1+s)^{3 / 2-2 \delta}} d s\right) \\
& \leq \frac{1}{(1+t)^{1 / 2}}\left(C C_{\mathrm{data}} \varepsilon+\check{C}(K \varepsilon)^{3}\right) \\
& \leq \frac{K \varepsilon}{2(1+t)^{1 / 2}},
\end{aligned}
$$

provided $K$ is large and $\varepsilon$ is small. As the estimate for $N_{2}(t)$ implies the desired estimate for $N_{1}(t)$, we have that for all finite intervals $[0, T)$, the estimates (4.8) imply that better estimates hold, thus concluding the proof.

\section{CONCluding REMARKS}

The analysis of timelike minimal surfaces and submanifolds introduces a family of geometrically-motivated quasilinear PDE systems which are both intriguing and relatively unstudied. The work done by Brendle and Lindblad and continued here constitutes merely a first step in this analysis.

Among other things, one would hope to see progress on the characterization of local well-posedness for the timelike minimal submanifold PDEs in general Lorentz spaces, as well as discovery of nontrivial stable solutions other than the flat planes one finds in Minkowski space. Exploring the nature and formation of singularities in these submanifolds should lead to interesting new phenomena.

Further, it is interesting to explore the relation between the timelike minimal surface PDE's and the classical minimal surface problem, see e.g. [LW] and references therein.

In addition to the case considered in this paper, the more general $D$-brane equations which arise when various matter fields are incorporated into the action occur in string theory and are of current interest in theoretical physics. It is clear that timelike minimal submanifolds will provide a rich source of new mathematical problems for some time to come.

Acknowledgements. We thank Mark Keel and Igor Rodnianski for useful discussions. We thank the MFO at Oberwolfach and the Isaac Newton Institute in Cambridge for providing stimulating places in which to collaborate on this research. This work is partially supported by NSF grants PHY-0354659 at Oregon, and DMS0407732 at the University of Miami.

\section{REFERENCES}

[B] Brendle, Simon, Hypersurfaces in Minkowski space with vanishing mean curvature, Comm. Pure Appl. Math., 55, (2002), 1249-1279. 
[H1] Hörmander, Lars, Lectures on nonlinear hyperbolic differential equations, Mathématiques \& Applications 26, Springer-Verlag, 1997.

[H2] Hörmander, Lars, $L^{1}, L^{\infty}$ estimates for the wave operator, Analyse mathématique et applications, 1988, 211-234.

[KSS] Keel, Markus and Smith, Hart F. and Sogge, Christopher D., Almost global existence for quasilinear wave equations in three space dimensions, J. Amer. Math. Soc., 17, 2004, 109153 (electronic).

[K1] Klainerman, Sergiu, Weighted $L^{\infty}$ and $L^{1}$ estimates for solutions to the classical wave equation in three space dimensions, Comm. Pure Appl. Math., 37, 1984, 269-288.

[K2] Klainerman, Sergiu, The null condition and global existence to nonlinear wave equations, Lectures in Applied Mathematics, Vol. 23, Amer. Math. Soc., 1986, pp. 293-326.

[K3] Klainerman, Sergiu, Long time behaviour of solutions to nonlinear wave equations, Proc. Int. Congr. Math. (Warsaw, 1983), pp. 1209-1215.

[L1] Lindblad, Hans, A remark on global existence for small initial data of the minimal surface equation in Minkowskian space time, Proc. Amer. Math. Soc., 132, 2004, 1095-1102 (electronic).

[L2] On the lifespan of solutions of nonlinear wave equations with small initial data, Comm. Pure Appl. Math., 43, 1990, 445-472.

[LW] Senchun Lin and Tilla Weinstein, A better conformal Bernstein's theorem, Geom. Dedicata 81 (2000), no. 1-3, 53-59.

[S] Sogge, Christopher D., Lectures on nonlinear wave equations, Monographs in Analysis, II, International Press, 1995.

Department of Mathematics, University of Oregon, Eugene, OR 97403, USA

E-mail address: pallen1@math.uoregon.edu

AEI, Max Planck Institute for Gravitational Physics, D-14476 Potsdam, Germany and Department of Mathematics, University of Miami, Coral Gables, FL 33124, USA

E-mail address: andersson@aei.mpg.de

Department of Mathematics, University of Oregon, Eugene, OR 97403, USA

E-mail address: jim@newton.uoregon.edu 